\title{
Contribution of components of Green Supply Chain Execution- Marketing in Green Supply Chain Performance measurement-A Pilot Empirical Study of the Indian Automobile Manufacturing Sector
}

\author{
Mohd. Asif Gandhi \\ School of Engineering and Technology \\ Anjuman-I-Islam's Kalsekar Technical Campus \\ Affiliated to University of Mumbai \\ Panvel, Maharashtra, India
}

\begin{abstract}
This paper is one of the several extensions of the research works done by [5]. Green Supply Chain Practices have been known to have an impact on Green Supply Chain Performance [5].This paper tests empirically through a pilot study of the Indian Automobile Manufacturing Sector, the contribution of the four variables constituting the construct Green Supply Chain Execution-Marketing in Green Supply Chain Performance measurement. Also the paper establishes the reliability of the questionnaire instrument developed previously for measuring the construct Green Supply Chain Execution-Marketing and also for measuring the reliability of the four variables that constitute the construct Green Supply Chain Execution-Marketing. Further the paper establishes the correlation among these four variables. Finally this paper conducts Confirmatory Factor Analysis (CFA) to arrive at a single factor (linear combination of four variables constituting the construct Green Supply Chain ExecutionMarketing) to aid in measuring the construct Green Supply Chain Execution-Marketing. Finally the paper establishes the order of contribution of the four variables constituting the construct Green Supply Chain Execution-Marketing.
\end{abstract}

Keywords: Automobile; CFA; Green Supply Chain Execution-Marketing; Green Supply Chain Performance; Green Supply Chain Practices, Indian, Manufacturing Sector; Pilot Study

INTRODUCTION

Green Supply Chain Execution-Marketing has been identified as one of the ten Green Supply Chain Performance measures which are impacted by five Green Supply Chain Practices [5]. Accordingly, this paper identifies the variables constituting the construct Green Supply Chain Execution-Marketing [5]. Green Supply Chain Execution-Marketing in turn is a sub-construct of the main construct Green Supply Chain Performance. Since Green Supply Chain ExecutionMarketing has been identified as being constituted of four variables, it is of interest to know how these four variables fare in the pilot empirical study of the Indian automobile manufacturing sector by means of a questionnaire instrument [5]. It is also of interest to know the order of contribution of these four variables constituting the construct Green Supply Chain Execution-Marketing. The 50 automobile manufacturing plants that were surveyed during the pilot empirical study are among the ones listed in [2]. The survey methodology was used in line with the findings of [3].

\section{THE RESEARCH QUESTIONS}

The six research questions that have been addressed by this research paper are as follows: 
Research Question 1. To have a feel of the responses of the Indian Automobile Manufacturing Sector pertaining to the four variables constituting the construct Green Supply Chain Execution-Marketing.

Research Question 2. To know the reliability of the questionnaire instrument for measuring the construct Green Supply Chain Execution-Marketing.

Research Question 3. To know the reliability of the questionnaire instrument for measuring the four variables constituting the construct Green Supply Chain Execution-Marketing.

Research Question 4. How are the four variables constituting the construct Green Supply Chain Execution-Marketing correlated?

Research Question 5. How many factors are retained by the four variables constituting the construct Green Supply Chain Execution-Marketing?

Research Question 6. What is the order of contribution of the four variables constituting the construct Green Supply Chain Execution-Marketing?

\section{THE CONSTRUCT GREEN SUPPLY CHAIN EXECUTION-MARKETING AND ITS FOUR VARIABLES USED IN THE STUDY}

There are four variables that constitute the construct Green Supply Chain ExecutionMarketing. They are depicted in Table 1 in their abbreviated form.

Table 1. The four variables constituting the constituting the construct Green Supply Chain Execution-Marketing

The four variables constituting the construct Green Supply Chain Execution-Marketing

\begin{tabular}{|l|l|l|l|} 
GSCEXMARK1 & GSCEXMARK2 & GSCEXMARK3 & GSCEXMARK4 \\
\hline
\end{tabular}

\section{DESCRIPTIVE STATISTICS OF THE DATA ON GREEN SUPPLY CHAIN EXECUTION- MARKETING THAT WAS SCALED}

A five point balanced Likert scale was used to scale the data from respondents on whom a questionnaire was administered. The respondents were employees of Indian automobile manufacturing firms and /or their plants as mentioned in [2]. The data collected revealed the following descriptive statistics of the four variables constituting the construct Green Supply Chain Execution-Marketing.

Table 2. Descriptive Statistics of the data scaled by the questionnaire on Green Supply Chain Execution-Marketing

\begin{tabular}{|c|c|c|c|c|c|c|}
\hline \multicolumn{7}{|c|}{ Simple Statistics } \\
\hline Variable & $\mathrm{N}$ & Mean & Std Dev & Sum & Minimum & Maximum \\
\hline GSCEXMARK1 & 50 & 50 & 3.76000 & 1.11685 & 188.00000 & 1.00000 \\
\hline GSCEXMARK2 & 50 & 50 & 3.92000 & 0.98644 & 196.00000 & 1.00000 \\
\hline GSCEXMARK3 & 50 & 50 & 3.70000 & 1.18235 & 185.00000 & 1.00000 \\
\hline GSCEXMARK4 & 50 & 50 & 3.70000 & 1.18235 & 185.00000 & 1.00000 \\
\hline
\end{tabular}




\section{THE RELIABILITY OF THE INSTRUMENT FOR THE CONSTRUCT GREEN SUPPLY CHAIN EXECUTION-MARKETING}

The reliability of the questionnaire instrument developed by [5] for the construct Green Supply Chain Execution-Marketing is shown in Table 3 as 0.968858 which is considered to be an indicator of excellent internal consistency reliability [4].

Table 3. Reliability by Cronbach's Coefficient Alpha for the construct Green Supply Chain Execution-Marketing

\begin{tabular}{|c|c|}
\hline \multicolumn{2}{|c|}{ Cronbach Coefficient Alpha } \\
\hline Variables & Alpha \\
\hline Raw & 0.969056 \\
\hline Standardized & 0.968858 \\
\hline
\end{tabular}

The reliability of the questionnaire for the four variables that constitute the construct Green Supply Chain Execution-Marketing is shown in Table 4. All the four variables in Table 4 namely GSCEXMARK1, GSCEXMARK2, GSCEXMARK3 and GSCEXMARK4 have a reliability greater than 0.9 but less than 1 which is considered to be an excellent indicator of internal consistency reliability [4].

Table 4. Reliability of the individual four variables constituting the construct Green Supply Chain Execution-Marketing

\begin{tabular}{|c|r|c|c|c|}
\hline \multicolumn{3}{|c|}{ Cronbach Coefficient Alpha with Deleted Variable } \\
\hline \multirow{2}{*}{$\begin{array}{c}\text { Deleted } \\
\text { Variable }\end{array}$} & \multicolumn{2}{|c|}{ Raw Variables } & \multicolumn{2}{c|}{ Standardized Variables } \\
\cline { 2 - 5 } & $\begin{array}{c}\text { Correlation } \\
\text { with Total }\end{array}$ & Alpha & $\begin{array}{c}\text { Correlation } \\
\text { with Total }\end{array}$ & Alpha \\
\hline GSCEXMARK1 & 0.904914 & 0.964054 & 0.899235 & 0.965278 \\
\hline GSCEXMARK2 & 0.830030 & 0.984707 & 0.828849 & 0.984765 \\
\hline GSCEXMARK3 & 0.984326 & 0.941023 & 0.981921 & 0.941560 \\
\hline GSCEXMARK4 & 0.984326 & 0.941023 & 0.981921 & 0.941560 \\
\hline
\end{tabular}

\section{PEARSON'S CORRELATION COEFFICIENT AMONG THE VARIABLES STUDIED}

The Pearson's Correlation coefficient between different pairs of variables that constitute the construct Green Supply Chain Execution-Marketing is shown in Table 5. Since the values of correlation coefficient are positive, it indicates that all the four variables that make up the construct Green Supply Chain Execution-Marketing are oriented towards the goal of Green Supply Chain Execution-Marketing in a unidirectional manner. Also all the values of correlation coefficient are above 0.7 but less than 1 . This is also an indicator of internal consistency reliability [4]. 


\begin{tabular}{|c|c|c|c|c|}
\hline \multicolumn{5}{|c|}{ Execution-Marketing } \\
\hline \multicolumn{5}{|c|}{$\begin{array}{l}\text { Pearson Correlation Coefficients, } \mathrm{N}=50 \\
\text { Prob }>|\mathrm{r}| \text { under H0: Rho }=0\end{array}$} \\
\hline & GSCEXMARK1 & GSCEXMARK2 & GSCEXMARK3 & GSCEXMARK4 \\
\hline GSCEXMARK1 & 1.00000 & $\begin{array}{l}0.74171 \\
<.0001\end{array}$ & $\begin{array}{l}0.93347 \\
<.0001\end{array}$ & $\begin{array}{l}0.93347 \\
<.0001\end{array}$ \\
\hline GSCEXMARK2 & $\begin{array}{c}0.74171 \\
<.0001\end{array}$ & 1.00000 & $\begin{array}{l}0.85390 \\
<.0001\end{array}$ & $\begin{array}{c}0.85390 \\
<.0001\end{array}$ \\
\hline GSCEXMARK3 & $\begin{array}{c}0.93347 \\
<.0001\end{array}$ & $\begin{array}{c}0.85390 \\
<.0001\end{array}$ & 1.00000 & $\begin{array}{l}1.00000 \\
<.0001\end{array}$ \\
\hline GSCEXMARK4 & $\begin{array}{c}0.93347 \\
<.0001\end{array}$ & $\begin{array}{c}0.85390 \\
<.0001\end{array}$ & $\begin{array}{c}1.00000 \\
<.0001\end{array}$ & 1.00000 \\
\hline
\end{tabular}

\section{CONFIRMATORY FACTOR ANALYSIS OF THE CONSTRUCT GREEN SUPPLY CHAIN EXECUTION-MARKETING}

Using a statistical analysis software called SAS 9.2, Confirmatory Factor Analysis (CFA) was conducted on the construct Green Supply Chain Execution-Marketing which consists of four variables namely GSCEXMARK1, GSCEXMARK2, GSCEXMARK3 and GSCEXMARK4. Principal Component method was used as the initial factor method. Accordingly the Eigenvalues were obtained as shown in the Table 6.

Table 6. Eigen values obtained by using Principal Components Method as the initial factor method.

\begin{tabular}{|c|c|c|c|c|}
\hline & Eigenvalue & Difference & Proportion & Cumulative \\
\hline 1 & 3.66387946 & 3.39141979 & 0.9160 & 0.9160 \\
\hline 2 & 0.27245968 & 0.20879882 & 0.0681 & 0.9841 \\
\hline 3 & 0.06366086 & 0.06366086 & 0.0159 & 1.0000 \\
\hline 4 & 0.00000000 & & 0.0000 & 1.0000 \\
\hline
\end{tabular}

An Eigen value indicates the relative importance of each factor in accounting for the particular set of variables being analyzed. From Table 6 it is clear that the first factor can explain 3.66387946 variables. No other factor in Table 6 can explain at least one variable. Hence only one factor will be retained by MINEIGEN criterion as the only factor as shown by the factor pattern of Table 7. The variance explained by this factor is 3.6638795 .

Table 3. Factor pattern obtained for the single factor retained by MINEIGEN criterion

\begin{tabular}{|c|c|}
\hline \multicolumn{2}{|c|}{ Factor Pattern } \\
\hline Variable & Factor1 \\
\hline GSCEXMARK1 & 0.94470 \\
\hline GSCEXMARK2 & 0.89839 \\
\hline GSCEXMARK3 & 0.99104 \\
\hline GSCEXMARK4 & 0.99104 \\
\hline
\end{tabular}

The final communality estimates for the four variables constituting the construct Green Supply Chain Execution-Marketing are shown in Table 8. 
Table 4.The final communality estimates for Green Supply Chain Execution-Marketing

\begin{tabular}{|r|r|r|r|}
\hline \multicolumn{4}{|c|}{ Final Communality Estimates: Total = 3.663879 } \\
\hline GSCEXMARK1 & GSCEXMARK2 & GSCEXMARK3 & GSCEXMARK4 \\
\hline 0.89245138 & 0.80709696 & 0.98216557 & 0.98216557 \\
\hline
\end{tabular}

Communality estimates are indicative of how much of each variable is accounted for by the underlying factors taken together. A high value of communality means that not much of the variable is left over after whatever the factors represent is taken into consideration. In short the communality estimates are indicative of the relative contribution of each of the variables in the construct. Accordingly Figure 1 shows in the descending order, the relative contribution of each of the four variables of the construct Green Supply Chain Execution-Marketing as follows: GSCEXMARK3 and GSCEXMARK4 at the same level followed by GSCEXMARK1 and GSCEXMARK2.

\section{Communality Estimates of Green Supply Chain Execution-Marketing}

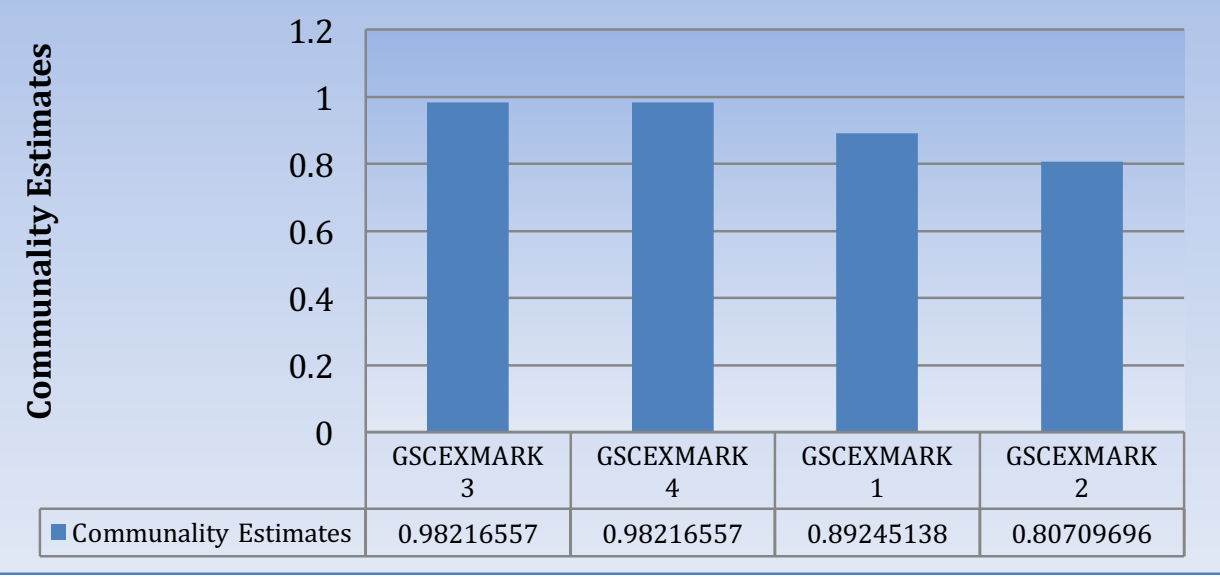

Figure 1. Contribution of the four components of Green Supply Chain Execution-Marketing in descending order.

\section{CONCLUSION}

The aim of this paper was to study the contribution of the four variables constituting the construct Green Supply Chain Execution-Marketing as a component measure of Green Supply Chain Performance. It was found that all the four variables in the study were strongly correlated with each of the other variables meaning that all the four variables involved are strongly oriented towards Green Supply Chain Execution-Marketing. The reliability of the construct Green Supply Chain Execution-Marketing was 0.968858 which is considered excellent. Also the reliability of the four variables constituting the construct Green Supply Chain Execution-Marketing was above 0.9 but less than 1 which means that the questionnaire is reliable to measure each of the four variables and also the construct Green Supply Chain Execution-Marketing as a whole. Also the results of Confirmatory Factor Analysis reveal that the single factor accounting for 3.6638795 variables is retained. In the descending order, the relative contribution of each of the four variables of the construct Green Supply Chain Execution-Marketing are as follows: GSCEXMARK3 and GSCEXMARK4 at the same level followed by GSCEXMARK1 and GSCEXMARK2. 


\section{References}

Emmett, S. and Sood, V., Green Supply Chains - An Action Manifesto. John Wiley \& Sons, 2010: p. 151-164.

Gandhi, M.A., A Review of the Indian Automobile Manufacturing Sector, IOSR Journal of Business and Management, 2017. 19(3), Ver II: p. 9-15. DOI: https://doi.org/10.9790/487X-1903020915.

Gandhi, M.A. and Sharma, S., A Review of Research Methodologies Linking Green Supply Chain Practices and Green Supply Chain Performance, International Journal of Supply Chain Management, 2014. 3(4).

George, D., and Mallery, M. Using SPSS for Windows step by step: a simple guide and reference, 2003.

Sharma, S., and Gandhi, M.A., Exploring correlations in components of green supply chain practices and green supply chain performance, Competitiveness Review, 2016. 26(3): p. 332-368.

DOI: https://doi.org/10.1108/CR-04-2015-0027. 